\title{
LÉGISLATION
}

\section{Concessions déclarées d'utilité publique et pose des conducteurs dans des terrains privés. Questions des indemnités. Décisions diverses.}

\author{
par Paul Bougault, Avocat à la Cour d'Appel de Lyon
}

\begin{abstract}
De nombreuses décisions ont été données récemment par divers tribunaux dans toutes les régions de la France sur l'interprétation de l'article 12 de la loi du 15 juin 1906. Notre collaborateur Paul Bougault a pensé que le meilleur moyen de faire connaître clairement l'impression qui se dégage de ces jugements, consistait non pas à donner simplement les textes, dont quelques-uns demandent les plus expresses réserves, mais à faire le sommaire des principes qui ont été admis par les juges et à grouper les solutions intervenues dans le même sens.
\end{abstract}

Sommaire des décisions rendues. - La déclaration d'utilité publique confere au concessionnaire le droit ou de provoquer l'expropriation d'une parcelle (dont il acquiert alors la propriété complète) ou de mettre en œuvre les servitudes spéciales créées à son profit par l'article 12 de la loi du 15 juin 1906. Mais l'établissement de ces servitudes ne peut être assimilé à une expropriation, puisque la loi a réservé au propriétaire de la parcelle le droit de demander l'enlèvement des conducteurs, s'il veut clore; surélever, bâtir. (Voir première décision ci-dessous : jugement du tribunal civil de Valenciennes, du 25 janvier 1928.)

Si la pose des engins cause un préjudice réel à la parcelle, et dans ce cas seulement, le propriétaire a le droit de réclamer devant le juge de paix une indemnité. (Voir première décision ; tribunal civil de Valenciennes et troisième décision; jugement du tribunal civil de Pau du 4 avril 1927.)

Cette indemnité doit être fixée en tenant compte du préjudice global estimé une fois pour toutes en ce qui regarde le présent et l'avenir ; elle ne doit pas donner lieu à une série d'indemnités annuelles, ce qui ferait ressembler la décision judiciaire à un contrat de bail. (Première décision, tribunal civil de Valenciennes.)

Une entreprise concédée soit avec la 'déclaration d'utilité publique, soit dans les conditions de subventions prévues par l'article 298 de la loi du 13 juillet 1925 est une entreprise de travail public. Elle a donc le droit de planter les poteaux dans les propriétés privées, et même, quand elle s'affranchit à tort des formalités préalables qui lui sont imposées par la loi et les règlements, on ne saurait dire qu'elles commettent sur une propriété privée une voie de fait qui serait justiciable du tribunal civil. La loi du 15 juin 1906 ayant confié à la justice de paix la fixation des indemnités qui peuvent ètre dues pour pénétration dans les propriétés privées, le tribunal civil doit renvoyer les parties litig antes à se pourvoir devant le juge de paix (deuxième décision : jugement du tribunal civil de Montluçon du 18 janvier 1929.)

Aucun texte ne contient l'obligation de faire fixer et à plus forte raison de payer l'indemnité préalablement à l'occupation. D'abord, la loi ne vise qu'une indemnité pouvant être due, s'il existe un préjudice ; par conséquent, il ne peut être question d'une indemnité dont le payement serait encouru dès que la pose dans telle ou telle parcelle serait décidée. Le juge de paix, en fait, se rendra un compte plus exact de l'indemnité à allouer, quand il statuera sur un établissement déjà accompli (troisième décision, jugement du tribunal civil de Pau.)
En exonérant de la servitude légale qu'il créait, les terrains clos, le législateur de 1906 a voulu désigner les terrains fermés de murs ou de clôtures équivalentes à des murs, c'est-à-dire présentant les mêmes conditions de solidité, d'élévation et de protection qu'un mur ordinaire, malgré la différence des matériaux employés. (Troisième décision, tribunal civil de Pau.)

Pour rendre légitime la demande d'indemnité pour occupation, le propriétaire doit prouver l'existence d'un préjudice matériel et certain : ne rentrent dans cette catégorie ni l'allégation que la pose d'un pylône aurait eu lieu en bordure d'un terrain à bâtir, ni que la présence des fils fait courir un danger aux personnes et aux animaux.

Il y a lieu d'allouer une indemnité fixée par un expert pour préjudice causé par le passage des ouvriers et le transport des matériaux dans le champ envisagé. (Quatrième décision; tribunal de paix de Bagnères-de-Bigorre du 23 août 1922.)

\section{PREMIËE DÉCISION}

\section{Jugement du tribunal civil de Valenciennes du 25 janvier 1928}

(Drusnes contre Etat français, Compagnie électrique

du Nord, Société de transport d'énergie électrique du Nord)

Le tribunal (1) : Attendu que l'article 12 de la loi du 15 juin 1906 sur les transports d'énergie électrique pose en principe le droit par les concessionnaires " d'établir à demeure des supports pour conducteurs aériens sur des terrains privés, non bâtis, qui ne sont pas fermés de murs ou de clôtures équivalentes ";

Attendu que ce mème article ajoute que cette implantation de supports n'entraîne aucune dépossession du propriétaire du sol, ne s'étend pas aux terrains bâtis ou clos de murs et ne fait pas obstacle au droit ultérieur de clôture et enfin, spécifie que " les indemnités qui pourraient être dues à raison des servitudes de passage seraient réglées en premier ressort par le juge de paix ;"

Attendu qu'il résulte de ce texte que la loi du 15 juin 1906 présente un caractère exceptionnel puisqu'elle grève la propriété d'une servitude légale et se substitue à l'application normale de la loi de 1841 sur l'expropriation, que le concessionnaire peut, d'ailleurs, faire jouer, s'il lui plaît, à ses lieu et place ;

(1) Ce jugement est reproduit par la Semaine juridique, année 1928 , page 1235 . 
Altendu que l'expression mème de servitude employée par le législateur indique qu'il y a un fonds servant grevé incontestablement d'une charge légale, que dès lors, pas plus que la servitude d'écoulement d'eau elle ne saurait, pour son seul établissement, donner lieu à aucune indemnité, bien que pouvant constituer une gène';

Attendu que cette solution s'impose d'autant plus que la loi a employé le mot " pourraient » qui indique, d'une façon précise, qu'il ne peut $y$ avoir lieu à indemnité obligatoire par le seul fail de l'implantation des pylônes sur le terrain grevé de la servitude d'appui sans constatation d'un préjudice spécial;

Attendu encore que la loi spécifie nettement qu'il ne s'agit que d'une simple dépossession, marquant ainsi à nouveau sa volonté d'exclure une indemnité obligatoire par le seul fait de l'exercice de la servitude.

Attendu d'autre part que ce caractère de simple dépossession est encore marqué par l'obligation du concessionnaire de ne pas poser de supports dans les terrains clos et bâtis et par la faculté laissée au propriètaire qui veut se clore ou bâtir, de demander le déplacement des supports d'appui qui pourraient entraver les constructions éventuelles.

Attendu, au surplus, que la loi ne prévoyant pas d'indemnites spéciales, il y a lieu, pour leur attribution, de se rapporter aux principes du droit commun en matière de travaux publics, dont toutes les obligations s'appliquent aux concessionnaires (art. $12, \S 1)$.

Attendu que l'indemnité due, dès lors, ne peut porter que sur le dommage direct, certain, actuel non simplemęnt éventuel, et qu'il résulte qu'on ne saurait envisager comme dommage certain une dépréciation de la valeur vénale ; mais que, néanmoins, l'indemnité doit comprendre tous les éléments découlant du dommage ainsi envisagé.

Attendu, en fait, que le jugement attaqué a donné comme mission à l'expert : $1^{\circ}$ estimer le préjudice causé par l'implantation et l'installation des pylônes (dégâts) ṕcur armer les pylônes et installer les lignes électriques, passage exceptionnel pour l'installation des dites lignes; $2^{\circ}$ estimer les indemnités dues pour les années écoulées depuis l'établissement des lignes électriques, pour réparer le préjudice causé : $a$ ) par l'existence desdits pylônes qui couvrent une partie des champs, rendent moins commode leur culture et entravent l'usage des machines agricoles ; b) par l'existence des sentiers de pylône à pylône ; $c$ ) par les travaux d'entretien, à raison des faits de passages, exercés pour l'entretien ou dégâts aux cultures dans les limites où ces faits ont causé un préjudice actuel matériel et certain.

Attendu que si le paragraphe I de la mission paraît conforme aux principes énumérés précédemment et n'est d'ailleurs pas contesté par les appelants, le paragraphe $2 a$ ) implique la reconnaissance d'une véritable redevance annuelle due chaque année d'une façon automatique et par le seul fait de l'implantation des pylônes, ce qui serait une interprétation erronée de la loi, ne tendant rien moins qu'à attacher une location annuelle à la charge d'une servitude légale;

Attendu que les indemnités dues pour la privation complète du sol occupé par la base du pylône et de la privation relative d'une certaine zone, en raison des difficultés à cultiver autour des pylônes, auraient dû être comprises avec les dégâts exceptionnels causés par l'installation proprement dite et calculée une fois pour toutes, en tenant compte de la valeur des terres et de la surface occupée; que la mission aurait dû être donnée dans ce sens, et qu'il y a lieu de ce chef de réformer le jugement attaqué ; que ces indemnités paragraphes 1 et 2 doivent être à la charge de l'Etat, constructeur et propriétaire des lignes électriques, et que les compagnies concessionnaires ne peuvent être inquiètées de ce chef;

En la forme : Attendu que le tribunal ne pouvant statuer définitivement par un seul et même jugement, il ne peul y avoir lieu à évocation conformément à l'article 473 du code de procédure civile et qu'il y a lieu de renvoyer devant le juge de paix compétent.

\section{Par ces motifs ;}

Le tribunal statuant en matière ordinaire, dernier ressort, infirme le jugement du tribunal de paix de Denain du 20 janvier 1927, dont est appel du chef du paragraphe $2 a$ ) de la mission donnce à l'expert;

Dit en conséquence que lexpert Lanthier devra completer sa mission et dire l'indemnité due une fois pour toutes, pour la privation complète du sol occupée par le pylòne et de la privation relative d'une certaine zone en raison des difficultés à cultiver autour des pylones tenant compte de la valeur des terres et de la surface occupée et en ayant soin d'exclure toute dépréciation de la valeur vénale des terres et en indiquant les bases de ces calculs : dit que ces indemnités s'ajouteront à celles prèvues par le paragraphe premier el seront supportées par l'titat propriétaire de la ligne. (MM. Franqueville, président; Hude, procureur de la République; Saint-Quentin, Deguerry, Doutriaux, avocats.)

\section{DEUXIÈME DÉCISION}

Jugement du tribunal civil de Montluson du 18 janvier 1929.

De Lavergnolles contre Compagnie électrique de la Loire et du Centre.

Altendu que de Lavergnolles Robert possède, commune de Saint-Vitte, une terre dite "Champ Prenat ", que la Compagnie électrique de la Loire et du Centre aurait implanté dans cet terre deux supports d'une ligne de transmission d'énergie électrique et que, pour l'aménagement de cette ligne, au-dessus de la propriété de Lavergnolles, la Compagnie électrique de la Loire et du Centre aurait gravement endommagé trois chènes;

Attendu que, qualifiant ces actes de "voies de fait " de Lavergnolles a assigné la Compagnie électrique de la Loire et du Centre pour faire judiciairement décider : $1^{\circ}$ que la dite compagnie devra supprimer ces supports et pour la partie qui surplombe la propriété de Lavergnolles, la ligne qu'ils soutiennent; $2^{\circ}$ qu'à défaut de cette suppression, il pourra y être procédé par de Lavergnolles ; $3^{\circ}$ que ce dernier sera indemnisé du préjudice résultant de ces travaux.

Attendu que la Compagnie électrique de la Loire et du Centre reçoit de l'Etat, du département du Cher et du Syndicat intercommunal de la région de Saint-Amand, une subvention dépassant les $10 \%$ de la valeur des travaux à exécuter pour l'électrification rurale de cette région; qu'en application des dispositions de l'article 298 de la loi du 13 juillet 1925, ensemble des dispositions des règlements d'administration publique des 27 et 31 décembre 1925, la Compagnie électrique de la Loire et du Centre doit être assimilée aux sociélés d'électrification ayant bénéficié d'une déclaration d'utilité publique.

Attendu que les dispositions de l'article 12 de la loi du 15 juin 1906 confèrent à ces sociétés le droit de " faire passer les conducteurs d'électricité au-dessus des propriétés privées... d'établir à demeure... des supports pour conducteurs aériens sur des terrains privés non bâtis et non fermés de murs ou de clôtures équivalentes ", comme "de couper les branches d'arbres 
se trouvant à proximnité des conducteurs aériens d'électricité "; que les tribunaux de l'ordre judiciaire sont incompétents lorsque la faute, génératrice du dommage à réparer se rattache, d'une manière indivisible comme dans le cas à l'exécution d'un travail public. (Cass. civ. divers arrêts : D. 1923, 1 ; 93.)

Attendu, il est vrai, que de Lavergnolles souligne qu'aux termes du même article 12 de la loi du 15 juin 1906, "l'exécution des travaux "permis au concessionnaire d'un service public de distribution d'énergie électrique " doit être précédée d'une notification directe aux intéressés, d'une enquête spéciale " et que cette exécution ne peut avoir lieu qu'après approbation par le préfet du projet de détail des tracés "; qu'aucune de ces formalités n'ayant été remplie au jour de l'exploit introductif d'instance, affirme-t-il, de Lavergnolles entend ignorer, quant aux travaux incriminés, toute concession et tout concessionnaire pour ne voir dans l'auteur de ces travaux qu'une société ordinaire, soumise aux règles de droit commun;

Mais, attendu que "le caractère de travail public, dépend de l'objet des travaux exécutés et non point de l'accomplissement des formalités qui doivent l'accompagner " (Cass. civ. 19 juillet 1922 et 25 avril 1923, Gaz. Pal. $1922,2.371$ et $1923,2.225$. D. 1926, 1.63) qu'ont le caractère de travaux publics " les travaux d'établissement du service public de distribution de l'énergie électrique exécutés en vertu d'un contrat de concession passé avec une commune ". (Cass. civ. 3 décembre 1923, Gaz.Pal. 1924,1234 D. 1926, 1.63.);

Attendu que pour avoir même avant le strict et total accomplissement des formalités, réalisé les actes à elle reprochés, et, du seul fait que ces actes se rattachent de manière indivisible à l'exécution d'un travail public " la Compagnie électrique de la Loire et du Centre n'a pas commis de voie de fait emportant violation d'un droit de propriété, que l'exécution de travaux de la nature de ceux faisant l'objet de la protestation de Robert de Lavergnolles n'entraine en effet aucune "dépossession " el que les indemnités qui " pourraient" être dues à raison de ces travaux doivent, aux termes de la loi du 15 juin 1906, être réglées en premier ressort par le juge de paix; qu'au surplus, et même sous l'empire de la législation antérieure à la loi du 13 juillet 1925 , des travaux publics exécutés avant la déclaration d'utilité publique, s'ils pouvaient être suspendus ne pouvaient être supprimés par décision d'un tribunal de l'ordre judiciaire (arrêt précité du 25 avril 1923$)$.

Par ces motifs, se déclare incompétent et renvoie de Lavergnolles devant qui de droit; condamne de Lavergnolles en tous les dépens. (M. Malo, proc. Rép. Mes Georges et Menut, av.)

\section{TROISIÈME DÉCISION}

\section{Jugement du tribunal civil de Pau du 4 avril 1927}

(Société des Forces motrices de la Vallée d'Aspe contre Bousquet.)

Attendu qu'à la date du 15 décembre 1923, une convention est intervenue entre l'Etat français et la Société des Forces motrices de la vallée d'Aspe pour la concession de lignes de transport d'énergie électrique à soixante mille volts, entre les Forges d'Abel à Bayonne et les Forges d'Abel à Laruns, que par décret en date du 6 mars 1924, cette convention a été approuvée et la concession déclarée d'utilité publique;

Attendu que le sieur Bousquet étant propriétaire des parcelles $345,316,311,315,314,300,5301,302,303,304$, 51, de la section $A$, du Plan cadastral, de la Commune de Bedous, lesquelles parcelles devaient être traversées par la ligne électrique la notification prescrite par l'article 12 de la loi du 15 juin 1906 lui furent faites directement, le 19 avril 1924, que le tracé des lignes a été approuvé par arrêté préfectoral en date du 5 juillet 1924.

Attendu que la Société des Forces motrices de la Vallée d'Aspe ayant voulu faire procéder sur la propriété du sieur Bousquet aux travaux nécessaires à l'établissement de la ligne des Forges d'Abel, à Bayonne, et notamment à l'installation des pylônes destinés à servir de supports aux conducteurs aériens du courant électrique, le dit sieur Bousquet s'y est opposé.

Que ce refus a motivé la demande formée à son encontre;

$1^{\circ}$ Attendu qu'aux termes de l'article 12 de la loi du 15 juin 1906, la déclaration d'utilité publique d'une ligne d'énergie électrique astreint de plein droit aux servitudes énumérées au dit article, les terrains qui doivent être traversés par la ligne que si ce texte prévoit le paiement aux propriétaires d'indemnités qui doivent être réglées en premier ressort par le juge de paix, il ne résulte nullement de ces termes que ce règlement doive être préalable à l'exécution des travaux;

Que, bien au contraire, l'expertise prévue par la loi en cas de désaccord suppose nécessairement que l'expert désigné par le juge de paix se trouvera, pour évaluer le dommage causé au fond par l'exercice des diverses servitudes, en présence de travaux complètement effectués; qu'enfin le principe de l'indemnité préalable, posé par l'article 545 du Code civil ne s'applique qu'au cas où il y a cession de propriété, ce qui n'existe pas en l'espèce ;

$2^{\circ}$ Attendu que le fait que les pylônes actuellement employés pour l'établissement des lignes électriques auraient une base d'implantation très supérieure à celle d'un simple poteau télégraphique ne saurait modifier le caractère de la servitude en vertu de laquelle ils sont installés;

Que dans l'un et lautre cas, le propriétaire du fonds grevé est privé de la jouissance d'une surface plus ou moins importante de son terrain, dont il reste cependant propriétaire.

Que la largeur de support implanté doit seulement entrer en ligne de compte pour l'évaluation de l'indemnité due.

$3^{\circ}$ Attendu qu'en édictant que l'établissement de canalisation ou de supports aériens ne pourraient avoir lieu, sur des terrains privés, que si ces terrains étaient non bâtis et non fermés de murs ou de clôtures équivalentes, le législateur a entendu seulement exonérer de la servitude les terrains qui, par suite des constructions ou des clottures qu'ils ont reçues se sont classés dans une catégorie vraiment différente des terres de culture et subiraient, du fait de cette servitude, un préjudice considérable.

Qu'en conséquence, par terrain bâti, il faut entendre celui qui touche soit à une habitation, soit à un établissement industriel ou commercial, soit à tous bâtiments analogues dont le dit terrain n'est que l'accessoire.

Et par terrain fermé de murs ou de clôtures équivalentes, le terrain qui est complètement enclos soit par un mur, soit par une clôture présentant des caractères de solidité d'élévation de protection comparables à ceux d'un mur bien que construit à l'aide de matériaux bien différents.

Attendu que si les parcelles ou certaines des parcelles appartenant au sieur Bousquet présentaient le caractère de terrains bâtis ou fermés de murs, la Société demanderesse devrait, en ce qui concerne ces parcelles, mettre en œuvre la procédure d'expropriation prévue par la loi du 3 mai 1841 ;

Mais, attendu que Bousquet déclare ne pas faire valoir ce moyen, se bornant à demander acte de ce qu'il se réserve de le faire valoir à l'avenir ; 
Altendu que c'est à tort que Bousquel prétend que la Société des Forces motrices n'a pas observé à son egard les formalités prescrites au concessionnaire par l'article 12 de la loi du 15 juin 1906 en ne lui faisant pas connaître le point exact où seront implantés les pylônes dans sa propriété.

Que le fait qu'il invoque à l'appui de cette prétention, à savoir que, lors de l'installation d'une première ligne, la Société demanderesse aurait fait subir une déviation au tracé projeté, en lui faisant emprunter une partie du tracé de la deuxième ligne faisant l'objet du procès actuel n'est pas de nature à créer à son préjudice l'incertitude dont il argue.

Que le sieur Bousquet en droit d'exiger que le second tracé soit respecté, comme il eût été en droit d'exiger que fut respecté le tracé de la première ligne.

Qu'à ce sujet, la Société demanderesse qui soutient d'ailleurs n'avoir opéré la déviation à laquelle fait allusion le sieur Bousquet que sur sa demande et pour la commodité offre de rétablir la première ligne suivant le tracé prévu ;

Attendu qu'il échet de faire droit aux demandes de donner acte des parties;

Attendu qu'il n'y a pas lieu d'accueillir la demande de dommages-intérêts formée par les parties, celles-ci ne justifiant aucun préjudice actuel.

Mais, attendu qu'il est de principe que des dommages-intérêts à calculer par jour de retard sont dus de plano au créancier par le débiteur d'une obligation en cas de morosité.

Par ces motifs :

Le tribunal, jugeant publiquement en matière ordinaire et en premier ressort,

Après en avoir délibéré conformément à la loi ;

Rejetant toutes autres conclusions des parties;

Dit et juge que l'indemnité prévue par l'article 12 in fine de la loi du 15 juin 1906, n'est pas nécessairement préalable à l'exercice des servitudes instiluées par le dit article au profit du concessionnaire.

Dit et juge que la largeur de base des supports aériens ne saurait, en l'espèce, influencer sur le caractère de la servitude de laquelle ils sont établis.

Dit et juge que la Société des Forces motrices de la Vallée d'Aspe a observé à l'égard du sieur Bousquet, en ce qui concerne la notification, les formalités prescrites au concessionnaire par l'article 12 de la loi du 15 juin 1906.

Dit que dans les vingt-quatre heures de la signification du présent jugement, le sieur Bousquet sera tenu de laisser pénétrer sur sa propriété et notamment sur les parcelles 300 et 302 , section A du Plan cadastral de la commune de Bedous, la Société des Forces motrices de la Vallée d'Aspe ou ses préposés pour effectuer tous travaux nécessaires à l'établissement de la ligne de transport d'énergie électrique à soixante mille volts des Forges d'Abel à Bayonne et ce sous une astreinte de mille francs par chaque jour de retard.

Donne acte à Bousquet de ce qu'il se réserve formellement de faire valoir à l'avenir le moyen qu'il n'invoque pas aujourd'hui tiré de ce que sa propriété scrait bâtie enclose de murs.

Donne également acte à Bousquet de ce qu'il fait réserve expresse de tous ses droits en ce qui concerne la première ligne établie sur les parcelles lui appartenant;

Donne acte à la Société des Forces motrices de la Vallée d'Aspe de ce qu'elle entend se conformer à toutes les obligations résultant pour elle de la loi du 15 juin 1906, et notamment de payer toutes les indemnites pouvant être dues pour l'implantation des pylones et le passage en surplomb de la ligne aérienne dont il s'agit, ainsi que pour tous autres dommages résultant de travaux.

Donne également acte à la Société des Forces motrices de la Vallée d'Aspe de ce qu'elle déclare qu'elle est prête à rétablir la première ligne déjà installée sur le terrain de Bousquet, conformément au tracé primitif approuvé et à faire disparâtre la variante qui avait été demandée par Bousquet lui-mème en cours d'exécution des travaux.

Condamne le sieur Bousquet aux dépens.

(Hermann, président; Boyer, substitut du procureur de la République; Caramayor et Dufau, avocals.)

\section{QUATRIÈME DÉCISION}

Jugement du tribunal de paix de Bagnères-de-Bigorre, 23 aon̂t 1922.

Société de Lumière et Force des Pyrénées contre Dardignac.

Attendu que, par citation en date du 28 juillet 1922, le sieur Dardignac réclame à la Société Force et Lumière des Pyrénées diverses indemnités, savoir :

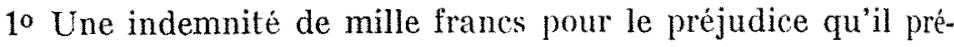
tend avoir subi du fait de la pose du pylône dans sa propriété, sise en bordure de la route nationale, sur le prolongement de la rue Maréchal-Pétain.

$2^{\circ}$ Une indemnité de deux cents franes pour le préjudice résultant de ce fait que la ligne aérienne traversant sa propriété crée en cet endroit, en cas de rupture de fil, un danger de mort pour les ouvriers et animaux qui pourraient s'en approcher;

$3^{\circ}$ Une indemnité de cinquante franes pour le dommage causé à une prairie pour le passage des ouvriers et des voitures lors de l'installation du Pylône;

Attendu que, par décret du 5 mars 1921, la Société Force et Lumière des Pyrénées a été déclarée d'utilité publique, a utilisé les terrains particuliers pour le support et le passage des fils conducteurs de l'électricité entre Gripp et Tarbes; que ce décret a été rendu en exécution de la loi du 15 juin 1906 sur les distributions d'énergie électrique, laquelle n'accorde aucune indemnité aux propriétaires assujettis aux servitudes; qu'elle prévoit, sauf en cas de dommage occasionné à leurs fonds ; qu'il en découle nécessairement, pour le concessionnaire, le droit de faire passer ses ouvriers sur les propriétés particulières grevées de ces servitudes.

Que, toutefois, si ce passage causait un dommage quelconque au propriétaire, le concessionnaire serait tenu de réparer ce dommage ;

Attendu que, d'autre part, qu'aux termes du dernier paragraphe du même article 12 , les juges de paix sont compétents pour statuer en premier ressort sur les indemnitès qui peuvent être dues aux propriétaires à raison des dites servitudes d'appui, de passage ou d'ébranchage prévues par la dite loi.

Attendu que, qur les premier et deuxième chefs de réclamalion, le demandeur ne justifie d'aucun dommage ; qu'il prétend simplement d'une part, que l'emplacement sur lequel est posé le pylône est un terrain à bâtir, lequel se trouve désormais impropre à la construction et que, d'autre part, la ligne aérienne qui traverse sur une longueur de trente-neuf mètres sa propriétí en nature de foin, présente à cel endroit un danger sérieux pour les ouvriers et les animaux. 
Attendu, sur ce point, qu'il apparaîl des termes de la loi lu 15 juin 1906 que les propriétaires du terrain utilisé pour 'emplacement du pylône et pour le passage des fils électriques le peut réclamer une indemnité que si un dommage actuel t certain, et non éventuel, lui a été causé soit par la pose des ylônes, soit par le passage des ouvriers, soit par l'exercice des iervitudes établies par la loi : qu'aucune indemnité ne lui est tue si cet exercice ne lui a causé aucun préjudice car la loi ne révoit au profit du propriétaire aucune indemnité pour l'établissement et l'exercice des serviludes dont elle grève les terrains sur lesquels sont conduits les fils électriques; que, dans ces conditions et quant à ces deux chefs de réclamation, le sieur Dardignac ne saurait prétendre à une indemnité ;

Attendu toutefois que la Société défenderesse a déclaré à notre audience faire offre au sieur Dardignac de la somme de cinquante francs pour la pose du pylône, plus vingt-cinq centimes par mètre courant de ligne ; qu'il y a lieu de donner acte à la dite Société de l'offre ainsi faite à notre barre;

En ce qui concerne le troisième chef de réclamation :

Attendu que, sans contester la matérialité du dommage, la Société défenderesse soutient que la somme réclamée est notablement exagérée et allègue que le préjudice causé est insignifiant, que les parties ont été d'accord pour recourir à une expertise aux fins d'évaluation du dommage ; que cette mesure paraît nécessaire pour éclairer le tribunal qui n'a aucun élément d'appréciation qu'il convient de confier cette mission au sieur Parade Gervais, expert, demeurant à Aste, canton de Campan, arrondissement de Bagnères-de-Bigorre.
I'ar ces molifs : Nous rejetons, comme non justiliée, la demande en indemnité formée par M. Dardignac en ce qui touche les premier et deuxième chefs de réclamation, donnons acte à la Société défenderesse de l'offre faite par elle à notre barre de payer la somme de cinquante franes pour l'installation du pylône, et, en plus de vingt-cinq centimes par mètre courant de ligne aérienne, et, avant dire droit sur le troisième chef de réclamation, tous moyens de conclusions des parties demeurant réservées, nommons, à titre d'expert, le sieur Parade Gervais, demeurant à Aste, avec mission de se transporter sur les lieux, d'examiner, en présence des parties, ou elles dûment appelées, les dommages causés à la prairie du demandeur par suite du passage des ouvriers et des voitures, lors de l'installation du pylône et de la ligne, de les évaluer en s'entourant de tous renseignements utiles, concilier les parties si faire se peut, et, à défaut de conciliation, rédiger un rapport dans lequel il formulera son avis motivé, rapport qu'il devra déposer au greffe de cette justice de paix dans un délai d'un mois ; continuons la cause à notre audience du 29 septembre prochain, parties intimées d'être présentes, dépens réservés.

Ainsi jugé et prononcé en audience publique, au prétoire de la justice de paix du canton de Bagnères-de-Bigorre, les jours, mois et an que dessus, 25 août 1922, par nous, Aurélien Bouny, juge de paix du canton de Bagnères-de-Bigorre, arrondissement dudit département des Hautes-Pyrénées, assisté de M. Guillaume Braca, greffier de la justice de paix du canton de Bagnères-de-Bigorre. 